\title{
The Phoenix Fire Model for Jet Creation: The Role of Magnetic Fields in the Production and Propagation of Relativistic Jets
}

\author{
David L. Meier ${ }^{1, a}$ \\ ${ }^{1}$ California Institute of Technology
}

\begin{abstract}
Using recent observational and theoretical results, I outline the latest ideas on how relativistic jets are produced by rotating magnetic fields, along with the physical processes that result in the FR I/II dichotomy. Simply put, at the end of the acceleration and collimation phase the now super-magnetosonic jet (the "Phoenix") passes through a master recollimation shock (the "fire") and is reborn as a new and more stable collimated MHD flow. Depending on whether the magnetic dissipation in the shock is negligible or substantial, the final jet will be either trans-magnetosonic (a BL Lac/FR I source) or super-(magneto)sonic with a weak magnetic field (an FSRQ/FR II source). Predictions are made about the behavior of additional observational and theoretical studies that can test this hypothesis more completely.
\end{abstract}

\section{Motivation: The Fanaroff and Riley Class Division Puzzle}

\subsection{Kiloparsec-scale Clues to its Physical Origin}

The Fanaroff and Riley division of extragalactic radio source morphologies into two distinct classes identifies FR I sources as those with bright radio emission near the jet origin or "core" (galactic center or quasar) and diffuse emission far from the origin, while FR IIs are those with bright emission far from the jet origin and diffuse emission closer to the core. Furthermore, the FR Is are identified with less luminous radio sources and the FR IIs with the most powerful sources observed [9].

Understanding the physical processes that produce this division has long been a vexing puzzle in this field. Bicknell [3] argued that FR II sources are those that propagate through the interstellar medium (ISM) at speeds greater than the jet internal sound speed, while FR Is propagate at trans-sonic speeds that lead to an eventual slowing of the jet to subsonic flow. Meier [25], on the other hand, argued that processes near the black hole (BH), where the jets are produced, could result in jets being ejected with very high speeds if the jet-production power were high or with slower speeds if the power fell below a "magnetic switch" threshold. However, the discovery of sources with hybrid FR I/II morphology or "HYMORs" (one jet with FR I structure and a counterjet with FR II structure) [13] essentially eliminated any scenario that assumes the FR $\mathrm{I} / \mathrm{II}$ division is rooted in processes very near the $\mathrm{BH}$ : the dynamical time at, say, $10 r_{S}$ (Schwarzschild radii) from a $10^{9} M_{\odot} \mathrm{BH}$ is of order a few days, and any asymmetry in jet production there probably would disappear on time scales of a month or so. HYMORs, on the other hand,

\footnotetext{
a e-mail: dlm@jpl.nasa.gov
}

clearly have existed in that asymmetric state for at least the jet propagation time from core to lobes $(1-10 \mathrm{Myr})$, making a near-BH origin of the FR division extremely unlikely. However, the dynamical time scale in a typical $10^{12} M_{\odot}$ elliptical galaxy is of order $50-100 \mathrm{Myr}$, making the Bicknell model more consistent with the existence of HYMORs.

Nevertheless, there are indications that the origin of the FR I/II break occurs on a kiloparsec-scale, or smaller, rather than on a multi-kpc scale. In M87 (an FR I) the jet is seen to accelerate from the core to a distance of order the stationary component HST-1 $(\sim 0.3 \mathrm{kpc}$ deprojected), whereupon the jet continually decelerates to eventual sub-luminal speeds [2]. Jets from somewhat lowermass BHs, therefore, might be expected to have their FR nature determined at a similar galactic radius of $r_{\mathrm{FR}} \sim$ $0.1 \mathrm{kpc}\left(\mathrm{M}_{\odot} / 10^{9} \mathrm{M}_{\odot}\right)$ from the BH or so. The dynamical time scale at this distance [26] is $\tau_{\mathrm{dyn}} \sim r_{\mathrm{FR}} / \sigma_{V} \approx 0.3$ Myr (where $\sigma_{V} \sim 340 \mathrm{~km} \mathrm{~s}^{-1}$ is the velocity dispersion of the stars in a $10^{12} M_{\odot}$ galaxy). This is just the sort of minimum FR I/II origin distance that is still allowable by the HYMOR test (assuming ten dynamical times for a jet asymmetry to last in a galactic nucleus).

One final kpc-scale clue to the FR I/II division nature comes from measurements of the relative magnetic field strength in the outer jet compared to the equipartition value there (the threshold where the field itself is strong enough to affect or control the jet dynamics in the lobes). Werner et al. [39] used Spitzer data to pin down the infrared portion of the synchro-self-Compton spectrum of FR II hot spots and concluded that most have $B^{2} / B_{\mathrm{eq}}^{2}$ values well below unity, indicating that in FR II lobes the magnetic field plays essentially no dynamical role. This conclusion is consistent with that from early numerical simulations 
of jets (e.g., [31]), which were able to obtain FR II hot spot and lobe morphologies without including any effects of magnetic field dynamics. The FR I source M87, on the other hand, displays wiggles between 1 and $5 \mathrm{kpc}$ and strong Faraday rotation, indicating that helical magnetic fields exist there and that they may be influencing the dynamics of the flow [1].

\subsection{Parsec-scale Clues}

A number of widely-held "cherished beliefs" held by the AGN jet community are crucial to understanding the entire FR class division. These are as follows. Blazars are classified as flat spectrum radio quasars (FSRQs), lowfrequency BL Lacertae objects (LBLs or red blazars), and high-frequency BL Lacs (HBLs or blue blazars). All blazars are believed to be objects from a larger "parent" population that are seen at a small viewing angle to the jet axis. The parent population of FSRQs appears to be the FR II sources that have high-excitation nuclear emission lines (or class 'A' FR IIs), i.e. the FR II radio quasars; the parents of LBLs appear to be low-excitation (class 'B') FR IIs; and the parent population HBLs appears to be FR I sources (which are almost all class 'B' objects). These associations are determined by measuring largescale properties, such as low-frequency radio luminosity and optical/UV emission line properties of blazar sources, and comparing them with properties of FR I and II radio sources. (See, e.g., [12, 16].)

Parsec-scale jets in the different blazar classes have distinctly different magnetic polarization and component speed properties. (See, e.g., [10].) First, the apparent speeds of VLBI jets in FSRQs are systematically higher than those in BL Lacs, although there is some overlap between the two velocity distributions. This property correlates well with the identification these objects with the (supersonic) FR II and the (transonic) FR I sources, respectively, as described in the Bicknell model. However, BL Lac Lorentz factors tend to be around $3-5$ $\left(V_{j} / c \sim 0.94-0.98\right)$, while Bicknell deduced $\gamma_{j} \sim 1.2$ $-1.4\left(V_{j} / c \sim 0.6-0.7\right)$ for sources at the FR I/II break.

Secondly, the observed magnetic fields in FSRQs tend to be parallel to the jet axis; or, when moving components are modeled as shocks in the jet, the internal jet magnetic field is modeled as having a weak, random and tangled configuration $([14,15,23])$. On the other hand, the observed magnetic fields in BL Lac objects tend to be orthogonal to the jet direction; indeed, these orthogonal fields are likely to be indicative of an ordered helical magnetic field within the jet body, with the axes of the jet and helix coincident [11]. Again, these polarization results correlate well with the the kiloparsec properties of FR II and FR I sources, if the jet magnetic field in each is relatively weak and relatively strong, respectively - in terms of $B / B_{\text {eq }}$. It is very important for the reader to understand that, while the absolute magnitude of FSRQ magnetic fields may be greater than those in BL Lacs, we hypothesize here that the ratio $B / B_{\mathrm{eq}}$ in $B L$ Lacs is, in fact, greater than that in FSRQs. That is, it is suggested that FSRQs are plasma pressure dominated flows (like FR IIs), while BL Lac objects actually are magnetic dominated flows internally.

The above well-known results lead to a surprising, but unmistakable, conclusion. Somehow, extragalactic jets not only know early whether or not they are going to be an FR I or an FR II source (i.e., within only $10^{5-6} r_{S}$ of the black hole, they also have acquired morphological and magnetic properties that are similar to those of the kpc-scale jet they eventually will be. The origin of the FR class division, therefore, must lie deep in the nucleus of the host galaxy - within, say, $10-100 \mathrm{pc}$ or so of the central black hole. This is significantly closer (by a factor of more than ten) to the BH than envisioned in Bicknell's model.

Below I discuss theoretical and physical properties of accelerating magnetized jets that may naturally explain the origin and properties of the Fanaroff and Riley sequence.

\section{Important MHD Physics}

Understanding basic magnetohydrodynamics is as important to radio jet astronomers as understanding atmospheric physics is to stellar astronomers. The reason is that radio jets are, to a large extent, optically thin, allowing observers to view the inner kinematics and dynamics of magnetized jet flow. In this section I review the principles of MHD physics that potentially will be applicable to jet launching, acceleration, and propagation. To keep the discussion simple, I will deal mainly with non-relativistic flow expressions; relativistic ones are given in reference [26].

\subsection{MHD Waves}

MHD waves play the same role in magnetohydrodynamics as sound waves play in regular fluid flow. The difference is that there are three, rather than one, characteristic speeds: the Alfvén speed and two magneto-acoustic (the fast and slow) speeds.

\subsubsection{Basic MHD Wave Properties}

Sound Waves. The sound speed actually plays a role in MHD, so I will review that first; I will assume in this paper that the equation of state of the particle plasma is adiabatic, with an adiabatic index $\Gamma$. The expression for the sound phase speed (see reference [26]) is

$$
V_{p h, s}= \pm c_{s} \equiv \pm(\Gamma p / \rho)^{1 / 2}
$$

where $p$ is the ambient plasma pressure, and $\rho$ is the plasma mass density.

Alfvén Waves. Unlike sound waves, Alfvén waves are transverse and cannot travel normal to the magnetic field direction. The wave phase speed looks similar to that for $c_{s}$, but has a vector direction

$$
\begin{aligned}
V_{p h, A} & = \pm V_{A} \cos \chi= \pm \mathbf{k} \cdot \mathbf{V}_{A} / k \\
\mathbf{V}_{A} & \equiv \mathbf{B} /(4 \pi \rho)^{1 / 2}
\end{aligned}
$$

where $\chi$ is the angle between the propagation vector $\mathbf{k}$ and the magnetic field $\mathbf{B}$; and $V_{A}$ is the magnitude of $\mathbf{V}_{A}$. 
Magneto-Acoustic Waves. Like sound waves, the magneto-acoustic waves also are longitudinal, traveling in the direction of the compressional perturbation. The wave speeds are roots of a quartic equation, which actually is quadratic in the square of the wave phase speed. The four roots for the fast and slow speeds are

$$
\begin{aligned}
V_{F}^{2} & =\frac{1}{2}\left[c_{m s}^{2}+\left(c_{m s}^{4}-4 c_{s}^{2} V_{A}^{2} \cos ^{2} \chi\right)^{1 / 2}\right] \\
V_{S}^{2} & =\frac{1}{2}\left[c_{m s}^{2}-\left(c_{m s}^{4}-4 c_{s}^{2} V_{A}^{2} \cos ^{2} \chi\right)^{1 / 2}\right]
\end{aligned}
$$

where $c_{m s} \equiv\left(V_{A}^{2}+c_{s}^{2}\right)^{1 / 2}$ is the magnetosound speed.

\subsubsection{MHD Waves in Different Plasmas}

MHD waves are quite different depending on whether the energy density $U$ is dominated by the particle or magnetic internal energy.

MHD Waves in a Particle Dominated Plasma ( $U_{\text {part }}$ $>>U_{\text {mag }}$ or $c_{s}>>V_{A}$ ). When particle pressure forces dominate, the magnetic field will follow the flow, which can be turbulent (resulting in a tangled field) or laminar (resulting in a, perhaps sheared, field in the direction of the flow). This is a reasonable model for FSRQs [14, 23]. Alfvén and slow waves propagate along the field lines, but the wave path may be rather tortured and aimless. Fast-mode waves, on the other hand, can propagate in any direction at nearly the magnetosound speed, compressing both plasma and magnetic field in the direction of propagation. NOTE: many investigators refer to such waves as "sound" waves, but they really are magnetosound waves with their speed enhanced to nearly $c_{m s}$ - the RMS of both the sound and Alfvén speeds (equation 4).

MHD Waves in a Magnetically Dominated Plasma $\left(U_{\text {mag }}>>U_{\text {part }}\right.$ or $V_{A}>>c_{s}$ ). In a magnetically dominated plasma the magnetic field is ordered, and the plasma flows along field lines. As in weak-field plasmas, fast waves can propagate in any direction: normal to the field the wave speed is $c_{m s}$, and parallel to the field it is the Alfvén speed. As in a weak-field plasma, Alfvén waves can propagate along or skew to the field, but with a speed that decreases to zero when they try to propagate normal to the field. Slow waves also propagate along the magnetic field, but at the sound speed, or skew to it at an even slower speed, and of course with zero velocity normal to the field. Below I suggest that BL Lac sources are primarily magnetically dominated plasmas.

IMPORTANT NOTE: When $U_{\text {mag }} \sim U_{\text {part }}\left(V_{A} \sim c_{s}\right.$ or near-equipartition), all three types of wave (Alfvén, fast, and slow) will be important. Therefore, observers need to know the difference between a jet that has a magnetic field that is strong in a truly relative sense and dominates the jet internals ( $\left.U_{\text {mag }}>>U_{\text {part }}\right)$ and one that is strong in only an absolute sense (in $\mu \mathrm{G}$ ). A jet that may have a very strong magnetic field in $\mu \mathrm{G}$, but which is still weak compared to the overall jet internal dynamics, is not a magnetically dominated jet, no matter how large the field is. On the other hand, a jet that has a much smaller magnetic field (in $\mu \mathrm{G}$ ), but which is stronger than the other jet internal forces, is indeed magnetically dominated. And it is the relative importance of the magnetic field in a jet that will determine its overall observed properties, not the absolute strength of the field.

\subsection{MHD Jet Properties}

\subsubsection{The Magnetosonic Nature of Flowing Jets}

It is the thesis of this paper that most astrophysical jets, but particularly extragalactic radio jets, are MHD jets. That is, they are directed, collimated flows that are comprised internally of both particle plasma and magnetic field (in varying degrees of domination). Each MHD jet then has a definable magnetosound speed $c_{m s}$, which can be compared to the jet flow speed $V_{j}$; and the local magnetosonic property of a given jet is determined solely by the relationship between the jet speed and its internal magnetosound speed. Jets are classified, therefore, as being sub-magnetosonic $\left(V_{j}<<c_{m s}\right)$, trans-magnetosonic $\left(V_{j} \sim c_{m s}\right)$, or super-magnetosonic $\left(V_{j}>>c_{m s}\right)$, and this classification is independent of the relative strength of the internal particle and magnetic energy densities discussed above (i.e., independent of $V_{A} / c_{S}$ ).

\subsubsection{Types of Energy Transport in MHD Jets}

The introduction of a new internal plasma property (magnetic field, in addition to particle content) introduces not only new types of waves, it also introduces new types of plasma flows. MHD jets can vary in their properties depending on whether the energy flux is dominated by kinetic or magnetic (Poynting) transport. The relativistic kinetic energy flux is given by $F_{\text {kin }}=\gamma_{j}\left(\gamma_{j}-\right.$ 1) $\rho c^{2} V_{j}$ (where $\rho$ may include field and thermal mass densities, and $\gamma_{j}=\left(1-V_{j}^{2}\right)^{-1 / 2}$ is the jet Lorentz factor). This reduces to $F_{\text {kin }}=\rho V_{j}^{3} / 2$ in the non-relativistic limit, which we will invoke here for purposes of a simple discussion. The Poynting flux is given by an expression that depends on whether the jet rotational speed $\left(R_{j} \Omega_{j}\right)$ or its propagation speed $V_{j}$ is faster: $F_{\text {Poynt }}=$ $\left(B^{2} / 4 \pi\right) \cos \alpha \max \left(R_{j} \Omega_{j} \sin \alpha, V_{j}\right)$, where we now take the pitch angle to be $\alpha \sim 45$ degrees for simplicity. During jet acceleration $\left(V_{j}<V_{A}\right)$, the rotational Poynting flux dominates, but after the jet exceeds the supermagnetosound speed, the advection of magnetic flux dominates the Poynting flow.

Kinetic Dominated Jets $\left(\left[V_{A}^{2} \max \left(R_{j} \Omega_{j}, V_{j}\right)\right]^{1 / 3}<<\right.$ $V_{j}$ ). A kinetic dominated jet is propelled forward by the ram pressure of the plasma flow, with the energy flux being essentially $F_{\text {kin. }}$. An example of such a jet is the kiloparsec structure of Cygnus A and probably all other FR II sources. The morphology is similar to that seen in unmagnetized, supersonic jet simulations [20, 31], and the hot spots in the lobes have magnetic fields well below equipartition [39].

Poynting Dominated Jets $\left(\left[V_{A}^{2} \max \left(R_{j} \Omega_{j}, V_{j}\right)\right]^{1 / 3}\right.$ $\left.>>V_{j}\right)$. A Poynting dominated jet, on the other hand, is propelled forward by the screw action of a rotating torsional Alfvén wave "turbine" - the so-called barber-pole 
effect. In MHD the plasma cannot cross the rotating magnetic field lines, so the forward motion of the rotational wave must carry plasma in the direction of its propagation. Ampere's circuital law of electrodynamics shows that such a jet also will be "current-carrying", where the current flow is along the axis of the jet. An example of such a jet is the acceleration and collimation zone (ACZ), where jets are created by rotating black holes or rotating accretion flows. The kiloparsec-scale jet in M87, and many if not most other FR Is, is an example of a Poynting dominated jet, with its Faraday rotation gradients across the jet and possible helical kinks in the flow. I argue below that the VLBI jets in BL Lac sources (at least the HBLs that are linked to FR Is) also are Poynting dominated jets.

\subsubsection{MHD Waves and Shocks in MHD Jets}

We now are able to discuss the different types of MHD waves (and their shock counterparts) in the different type of MHD jets discussed above. It will be important to remember that both magneto-acoustic modes can generate shocks as well as simple waves, and both these phenomena will be longitudinal (producing a compression in the direction of the wave propagation). However, Alfvén waves are linear only and do not produce shocks. Furthermore, Alfvén waves will be transverse (producing a possibly visible perturbation in the plasma in the direction normal to the wave propagation direction). As above, I will discuss the kinetic dominated jet case first.

MHD waves in Particle and Kinetic Dominated Jets $\left(c_{s}>>V_{A}\right.$ and $\left.V_{j}>>\left[V_{A}^{2} \max \left(R_{j} \Omega_{j}, V_{j}\right)\right]^{1 / 3}\right)$. In this case the internal magnetic field is either tangled or sheared by the much stronger kinetic flow. This type of jet is generally applied to FSRQ and FR II jets [3, 14, 15, 23], with one exception: the term 'supersonic' is used, whereas, in fact, these jets should be called super-magnetosonic.

Alfvén waves and slow-mode waves and shocks in particle/kinetic-dominated jets will propagate along and skew to the weak, and possibly aimless, magnetic field (but not normal to it); their dissipation may be important to the the internal jet thermal properties but generally not to larger scale features that will be easily observable.

Fast-mode magneto-acoustic waves and shocks, on the other hand, will be strong and propagate in whatever direction they are launched - along, skew to, or even normal to the jet direction. They will compress both the jet plasma and the component of the magnetic field normal to the wave, enhancing the strength of the field in the plane of the wave. The speed of the fast-mode wave in the jet rest frame will be $c_{m s}$, which is a little larger than the sound speed $c_{s}$, and the speed of a propagating fast-mode shock can be $V_{\text {pattern }}>c_{m s} \gtrsim c_{s}$. In the observer's frame, the wave/shock speeds will be boosted by the jet Lorentz factor, of course.

MHD waves in Magnetic and Poynting Dominated Jets $\left(V_{A}>c_{s}\right.$ and $\left.\left[V_{A}^{2} \max \left(R_{j} \Omega_{j}, V_{j}\right)\right]^{1 / 3}>>V_{j}\right)$. In these jets the magnetic field is relatively much stronger $\left(V_{A} \sim c_{S}\right.$ or even $\left.V_{A}>>c_{S}\right)$ and has a helical structure, with the helix and jet axes aligned, that cannot be easily modified by plasma flow alone. Below I argue that this kind of jet should be applied to HBL and FR I jets, and this suggestion is in line with Gabuzda's conclusions on the role of helical magnetic field in BL Lac VLBI jets [10, 11]. I also argue below that HBL/FR I jets are only transmagnetosonic in nature (based on numerical simulations), and this concept is in line with the results of Bicknell [3], save for the fact that we now recognize these flows to be trans-magnetosonic rather than just trans-sonic.

Fast-mode magneto-acoustic waves and shocks in magnetic/Poynting-dominated jets will behave much like those in particle/kinetic-dominated ones, even though the underlying magnetic field is well ordered. Fast waves and shocks are able to compress both the plasma and magnetic field in the direction of the wave propagation, again enhancing the energy density of both and, therefore, the local radiation emitted. This longitudinal wave (or shock) would appear similar to the compressional longitudinal "slinky" wave (see, e.g., the image at http://www.geo.mtu.edu/UPSeis/images/Pwave_slinky.gif). The speed of the fast-mode wave in the jet rest frame will depend on the pitch of the magnetic field: $V_{F} \sim c_{m s}$ if the field is highly toroidal, while $V_{F} \sim V_{A}<c_{m s}$ if the helical field is quite open. However, the fast-mode speed always will be $\geq V_{A}$. The speed of a fast-mode shock can be much faster, but also will always be greater than the Alfvén speed.

As in particle/kinetic-dominated jets, Alfvén waves and slow-mode waves and shocks still will propagate along the magnetic field. While Alfvén waves could travel along the complete, circuitous helical field path, a possible sub-mode would be to travel as a largescale, transverse wave along only the longitudinal component of the magnetic field. Such a wave would appear very much like a transverse "slinky" wave (see, e.g., the image at http://www.geo.mtu.edu/UPSeis/images/Swave_slinky.gif). The speed of this longitudinal wave would be $\leq V_{A}$, nearly equal to $V_{A}$ if the helical field is open, and $<<V_{A}$ if the helix is highly toroidal.

Finally, slow-mode waves in a jet with a helical magnetic field would compress only the plasma particle component, leaving the magnetic field structure unchanged. They can propagate in the jet rest frame at the particle sound speed $c_{S}<V_{A}$ along the circuitous magnetic field. Again, a wave that propagates only in the direction of the jet/helix axis is possible, but at an even slower speed that would be proportional to the ratio of the ratio of the longitudinal magnetic field component $B_{Z}$ to the total magnetic field strength $B$. Of course, slow-mode shocks can travel faster than $c_{s}$, but it seems unlikely that slow-mode waves or shocks could propagate faster than an Alfvén wave along the jet body in any reasonable situation. Slow-mode waves/shocks in a magnetic/Poyntingdominated jet, therefore, may be very slowly-moving features in the jet rest frame whose observed speeds simply reflect the value of the jet Lorentz factor $\gamma_{j}$ itself. That is, slow-mode features may appear to be what used to be called "blobs". 

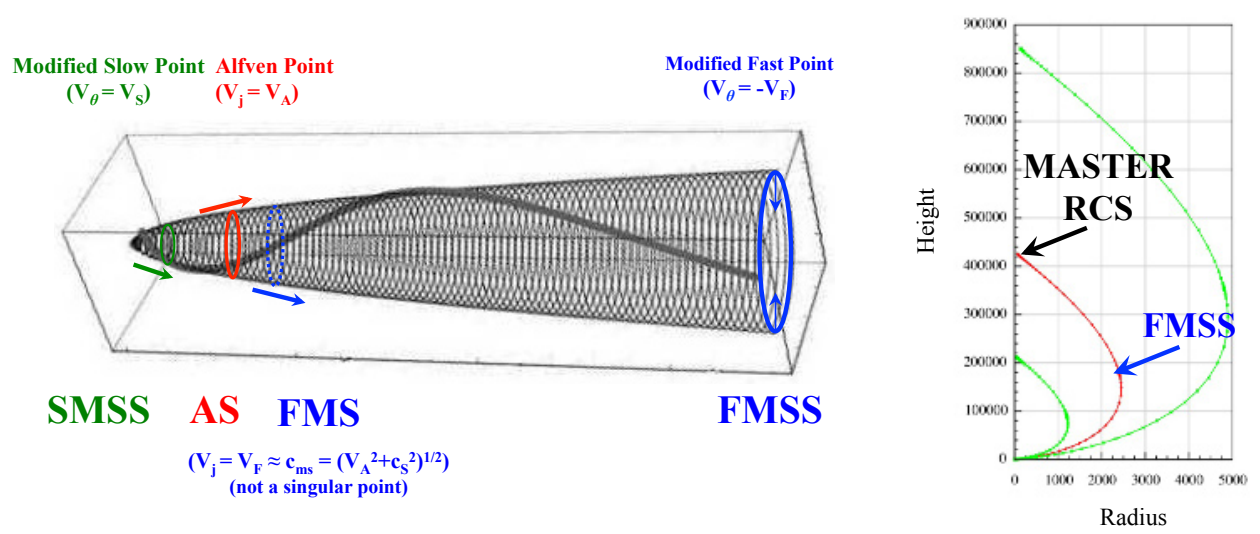

Figure 1. The Jet Acceleration and Collimation Zone. Left panel: acceleration and collimation from the launch point to the magnetosonic horizon (FMSS). Note the three singular/separatrix surfaces (SMSS, AS, and FMSS; see text for discussion); information flow via each associated MHD wave is only away from these surfaces. Also shown is the classical fast magnetosonic surface (FMS), where the flow speed of the jet exceeds the magnetosound speed, and the jet transitions from Poynting to kinetic energy domination. (Panel modified from one kindly provided by N. Vlahakis.) Right panel: three poloidal field/stream lines in a steady state, relativistic MHD model of an accelerating jet. (See Polko's work [34] for more details.) Note that, beyond the FMSS, the flow begins to over-collimate, crushing itself onto the jet axis in less than a magnetosound crossing time. This is likely to produce a strong shock, which we call the master recollimation shock. Note that the horizontal axis has a scale smaller than the vertical by a factor of $\sim 0.006$; this jet, therefore, is nearly 200 times longer than it is wide at its broadest point.

\section{Launching, Acceleration, and Collimation of MHD Jets}

\subsection{Jet Launching}

It is well known $[4,21,36]$ that it is difficult to launch a plasma outflow from the deep gravitational well that exists near the surface of an accretion disk with height $H<<R$. Here we define jet launching as a process that lifts the plasma from the vicinity of the disk surface to a height $Z \sim R$ where it can be accelerated upward and outward in a steadily diminishing gravitational potential. In this paper understanding the exact processes that launch jets from black hole systems is not of immediate importance. However, knowing the general classes of launching mechanisms is useful for the reader's further understanding of how important the various MHD wave phenomena are. One can classify the various means of jet launching into three categories.

Fast MHD Mode Launching. The process called the "magnetic tower" $[22,27]$ uses fast-mode MHD acoustic waves to launch jet material when the plasma is magnetically dominated. A helical magnetic field expands upward, carrying plasma with it.

Alfvén Mode Launching. The so-called BlandfordPayne process [4] uses a large-scale torsional Alfvén wave to centrifugally accelerate plasma outward and, like the magnetic tower above, eventually collimate and accelerate the plasma with a strong, upwardly expanding helical field. This also requires a dominant magnetic field.

Slow MHD Mode Launching. If the disk possesses a large corona with a strong magnetic field filled with hot plasma at, or above, the virial temperature, the thermal expansion of that plasma upward and outward along the field lines can be described as a slow MHD mode mechanism of jet launching. Beyond $Z \sim R$ the differential rotation of the magnetic field will create a magnetocentrifugal region and then a magnetic tower region beyond that, completing the creation of all the properties necessary to begin the jet acceleration and collimation process.

\subsection{Jet Acceleration and Collimation}

While almost all jet acceleration and collimation zones (ACZs) are below the resolution of astronomical imaging observations (including even VLBI in most cases), it is this region that has the greatest influence on the final nature of the jet that is dispatched from the central black hole system. We, therefore, will discuss what currently is known theoretically about this region and how it might appear when observed. Figure 1 shows the basic physical concepts of how jets are accelerated, collimated, and over-collimated toward a catastrophic collision on the jet axis.

\subsubsection{Critical Surfaces}

In steady state flow there exist "critical surfaces" where the flow across the surface passes from being below a characteristic group wave velocity to above that velocity. For our purposes the critical surfaces are not very important, but for reference these are the "cusp surface" or CS, "slow magnetosonic surface" (SMS), and "fast magnetosonic surface" (FMS). At these surfaces wave characteristics exist on one side of the surface (indicating some super-critical flow) and do not exist on the other side (subcritical flow). Note, however, that information can flow across critical surfaces in both directions via each associated MHD wave, so they do not indicate the places where magnetosonic horizons can form. 


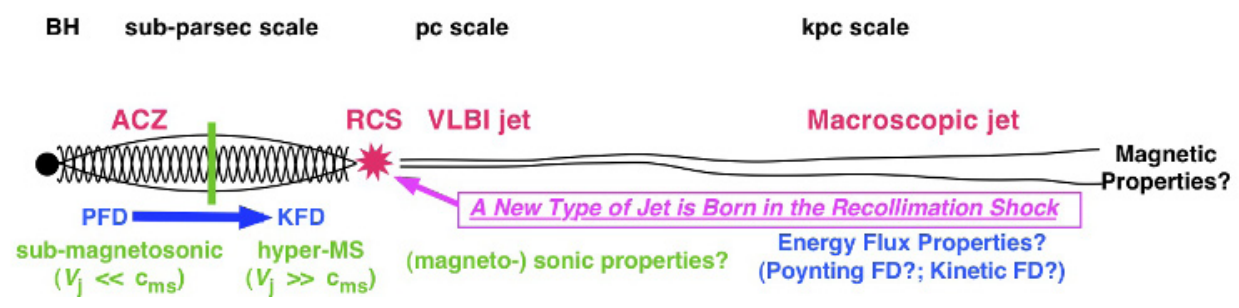

Figure 2. The Basic Phoenix Fire Hypothesis. Acceleration and collimation in the ACZ (see Figure 1) leads to a rather unstable, super-magnetosonic, kinetic-dominated jet that collapses and passes through a strong recollimation shock (RCS) - at the parsec scale or smaller. The jet then is reborn in the shock as a new and much more stable type, with properties that can be observed on the parsec scale with VLBI and on the kiloparsec scale with connected-element imaging radio interferometry.

\subsubsection{Separatrix Surfaces}

In a steady state there also exist "separatrix surfaces" where the direction of information flow normal to the surface is always away from that surface. These surfaces are of utmost importance to the structure of the plasma flow; in the MHD case there also are three: the "slow magnetosonic separatrix surface" or SMSS, "Alfvén surface" (AS), and "fast magnetosonic separatrix surface" (FMSS). In steady state numerical models these are singular surfaces, where the denominator of a wind equation equals zero along the surface, so the numerator also must equal zero on that surface in order to achieve a smooth plasma flow across it. Setting the wind equation numerator to zero adds an extra internal boundary condition that the flow must satisfy. In mathematical models where the wind equation has been reduced (using symmetry assumptions) to a one-dimensional equation, these surfaces become singular points along a representative streamline. In the MHD case the SMSS, AS, and FMSS then are often called the "modified slow point" (MSP), "Alfvén point" (AP), and the "modified fast point" (MFP).

Figure 1a shows an ACZ of a typical accelerating jet. The tight rotating helical magnetic field that both accelerates forward and collimates inward (toward the jet axis) is clearly visible. So are the MSP, AP, MFP locus rings of a streamline (heavy black line). The diagram is terminated where the MFP ring occurs.

The Magnetosonic Horizon. The FMSS surface (MFP locus) represents the place where information carried by MHD waves can travel only downstream. We sometimes call the FMSS the "magnetosonic horizon". In the jet flow beyond the FMSS no event or feature can affect the structure of the $A C Z$ via MHD waves. ${ }^{1}$ This property of the FMSS is crucial to the stability of the ACZ structure and to its ability to dispatch an MHD jet.

\subsubsection{Jet Properties at Different Stages of the ACZ Flow}

From several recent MHD steady state models of the jet ACZ [33-35, 37], which require that the flow pass through the FMSS/MFP, we now have a much better idea of

\footnotetext{
${ }^{1}$ Of course, electromagnetic radiation that travels at $c$ can send information upstream, but in a relativistic flow even this would be difficult.
}

how jets are accelerated from sub-magnetosonic to highly super-magnetosonic speeds by a rotating magnetic field in the relativistic regime as well as the non-relativistic.

ACZ Flow up to the Fast Magnetosonic Surface. Up to the FMS (not FMSS!) the jet remains Poynting dominated and the flow speed is sub-magnetosonic $\left(V_{j}<c_{m s}\right)$. The FMS lies just beyond the AS (Figure 1a); it occurs when the flow speed in the $\mathrm{Z}$ direction achieves the fast magneto-acoustic speed $V_{F}$ (which is only a little larger than $V_{A}$ ).

ACZ Flow Between the FMS and FMSS. Beyond the FMS the flow continues to be accelerated by the magnetic field, even though its speed is now super-magnetosonic. How can that be; the $Z$ gradient of the azimuthal field $\left(d B_{\phi}^{2} / d Z\right)$ is no longer effective in accelerating the jet forward after the flow speed exceeds $V_{F} \sim c_{m s}$ ? The answer is that the hoop stress of the azimuthal field $\left(B_{\phi}^{2} / R\right)$ is still quite strong and still collimates the jet far beyond the FMS. This accelerates the jet material toward the jet axis ( $-\theta$ direction), and because of the shape of the collimating jet, also (slowly) accelerates the jet forward - similar to squeezing a tube of toothpaste to producing outflow along the tube axis. The hoop stress remains effective in doing so until $\left|V_{\theta}\right|>V_{F}$.

ACZ Flow Beyond the Magnetosonic Horizon. Beyond the FMSS the flow is in a very interesting and unstable state. It is in hyper-magnetosonic flow along the jet axis. And it also is in a super-magnetosonic, overcollimating flow toward the jet axis. It will collide with itself on that axis within less than a magnetosound crossing time, making the flow unstable on a dynamical time scale to a catastrophic change in state. This overcollimating state is depicted in Figure 1b.

\subsection{The MASTER Recollimation Shock: The Fire in Which the Jet Phoenix is Reborn}

What will be the nature of this catastrophe? The likely outcome will be a strong MHD compression or shock at the jet constriction point that could significantly modify the flow. Figure 2 shows the main aspects of this hypothesis.

In a simple supersonic hydrodynamic flow [32] this is referred to as a "recollimation shock" or RCS. Generally (at least in simulations and laboratory experiments), these shocks are rather weak, with several forming after the first 


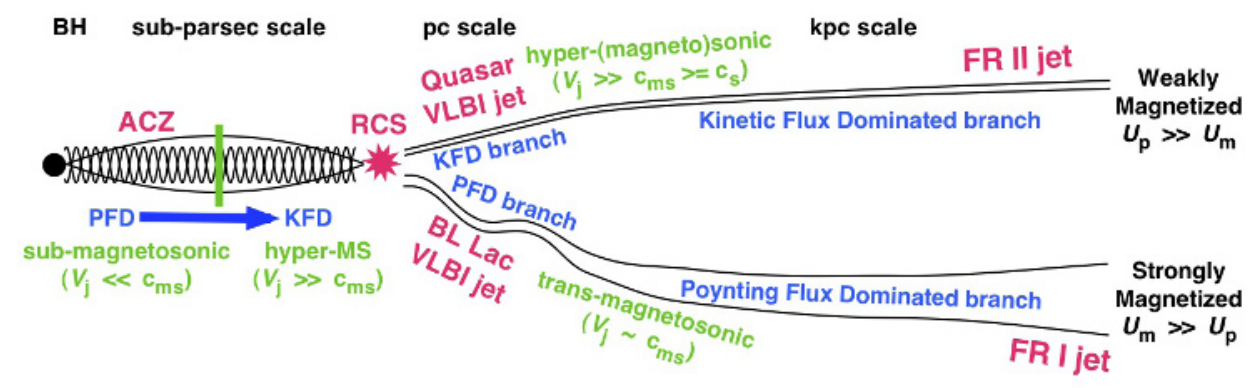

Figure 3. The Phoenix Fire Model for BL Lac/FR I and FSRQ/FR II Sources. (Note that the upper and lower branches indicate two different alternatives in Figure 2, not two simultaneous flows.) In the model, the flow beyond the recollimation shock is controlled by the dissipative processes in the RCS itself. Upper Branch: If the RCS is strong enough to dissipate the magnetic field, and radiate that internal energy, then the resulting flow will be weakly-magnetized and supersonic. (Formally, it will be super-magnetosonic, as it will carry a weak field which, together with the plasma pressure, will have a magnetosound speed slightly larger than the actual sound speed itself.) FR IIs and FSRQs show many properties of this kind of flow, including evidence of a weak magnetic field. Lower Branch: If the RCS is not strong enough to dissipate much of the internal magnetic field, then a magnetically dominated, trans-magnetosonic flow results (as occurred in simulations in references $[8,17,18,20]$ ). M87 and BL Lac jets show many properties of such a flow, including a possible helical magnetic field.

one as the jet "bounces", expands, overcollimates, and bounces again. Eventually, the internal energy of overcollimation/over expansion is dissipated, resulting in a steady supersonic flow downstream. In this case, the jet not only retains most of its forward momentum beyond the RCS, it also maintains its overall flow speed. However, if the shock is strong enough (as, e.g., a Mach disk in a jet hot spot) the conditions in the flow beyond the RCS could be quite different from the pre-shock ones. For example, the post-shock flow may be heated and significantly slowed compared to the pre-shock supersonic conditions.

In an overcollimating MHD jet the final post-shock properties have an even greater amount of parameter space they could occupy, because of the additional influence of the magnetic field. As above, a very strong Mach disk-like shock could cause the jet to be largely stopped, flowing laterally into a cocoon, or the jet could be allowed to flow forward, still strongly confined by the hoop stress of the azimuthal magnetic field. Furthermore, the internal properties (particle energy, magnetic field, etc.) could be significantly altered in the shock by processes like magnetic reconnection, energy dissipation, and radiative losses.

One crucial point is certain, however: no matter how catastrophic the RCS conditions are, they occur beyond the magnetosonic horizon (FMSS). So, the upstream steady state structure of the jet engine remains unaffected by what happens in the shock. The RCS only affects the jet structure downstream.

Because of the highly relativistic and supermagnetosonic nature of most jets in the nuclei of blazars, it seems highly likely that the result of the overcollimating jet at the end of the ACZ will be a single, very strong RCS. Here we call that the MASTER RCS. We also suggest that this singular feature will significantly alter the MHD flow into a (potentially more stable) final form that is, in fact, the jet that is observed as the extragalactic radio source. We call this assertion the Phoenix Fire Hypothesis (or $\mathrm{PhFH}$ for short) - the highly super-magnetosonic jet itself being the Phoenix that is reborn in the fire (the RCS) to become a BL Lac/FR I or FSRQ/FR II type of jet.

\section{The Post-Recollimation-Shock Jet: How FR I and II Sources are Born}

Below we discuss two possible scenarios for the post-RCS jet, one of which is a good model for FR I sources and the other is a suggestion for FR IIs. Both are displayed schematically in Figure 3.

\subsection{Jet Rebirth in a Moderately Strong Recollimation Shock}

Surprisingly, a significant amount of work has been formed on a possible model for producing FR I jets, although this application was not known at the time. In the mid-late 1980 s, shortly after the first simulations of hydrodynamic jets, several groups began to explore the behavior of MHD jets as well $[8,20]$. At that time it was numerically easiest to first examine jets with dominant toroidal magnetic field, so simulations were performed with different magnetic field strengths, all injected with the same sort of (magneto-)sonic Mach number. Ones with very weak fields $\left(U_{\text {mag }} / U_{\text {part }}<<1\right)$ behaved similarly to those with zero field $\left(U_{\text {mag }}=0\right)$ : they developed a strong bow shock as the jet propagated through the external medium, followed by a contact discontinuity that separated shocked external from shocked jet media. The hot shocked jet medium expanded laterally into a cocoon, and behind that, on the jet axis, was a strong Mach disk shock that terminated the supersonic jet flow.

As the field strength was increased, however, less of the jet material expanded into the cocoon and more was propelled forward in a "nose cone", which was actually a trans-(magneto-)sonic jet flow that was laterally confined by a still-strong toroidal magnetic field. See, e.g., Figs. 6, $11, \& 12$ of [20]. Although no longer super-magnetosonic, 
the speed of this new MHD jet was still fast - far faster than the advance speed of the Mach disk itself - so that, at least in the simulation, the constriction shock (Mach disk) was nearly stationary, while the new nose-cone jet became the dominant part of the entire flow structure. Furthermore (Figure 9 of [20]), the new jet contained multiple shocks along its axis, each created by the ejection of new material from a sort of magnetic chamber just beyond the Mach disk. Detailed analysis of the time-dependent behavior of this chamber (Figure 10 of [20]) showed that it periodically opened and closed on its downstream end, ejecting a "blob" of material every so often. As these simulations were two-dimensional, axisymmetric, this latter result needs to be taken with some caution until full 3-D simulations can be performed. (It is possible that the enforcement of axisymmetry limited the number of degrees of freedom the azimuthal field could direct the forces.)

This problem has been studied by other authors more recently $[17,18]$, and these results appear to be robust in the relativistic case as well, at least in 2-D axisymmetry. Full 3-D simulations of super-magnetosonic flows, their recollimation shocks, and the post-shock flow still need to be performed to confirm all the above details.

The bottom line is as follows: the "nose-cone" jet that results from passage of a super-magnetosonic jet through an RCS-like shock is a trans-magnetosonic MHD jet. With only one exception (that this jet is transmagnetosonic, not simply trans-sonic), this result is consistent with Bicknell's conclusion that FR I jets are transsonic flows that further decelerate as they propagate through the ISM and IGM. We, therefore, suggest here that FR Is sources are produced by black hole systems whose acceleration and collimation regions terminate in a moderately-strong RCS.

\subsection{Speculation on Jet Rebirth in a Very Strong Recollimation Shock}

To this author's knowledge, simulations that would address the formation of super-sonic FR II-type jets from an initially super-magnetosonic, MHD jet have yet to be performed. This is, of course, an exceedingly important problem, as it has the potential for answering the following crucial question: if extragalactic jets are all accelerated and collimated by strong, helical magnetic fields, why is it that the most powerful of these sources (FR IIs) are observed to have magnetic fields that are relatively the weakest - i.e., the internal fields are dynamically unimportant? Below I describe a plausible scenario in which this paradoxical behavior could arise in the context of the Phoenix Fire Hypothesis, and I suggest a series of MHD simulations that could test this scenario.

For sources with a high enough Lorentz factor (i.e., $\left.\gamma_{j}>>\gamma_{A} \equiv 1 /\left(1-V_{A}^{2}\right)^{1 / 2}\right)$ shear in the flow into the RCS might generate MHD turbulence. This, then would enhance the probability that the magnetic field could be torn, reconnect, and dissipate much of its energy into internal jet particle energy. When this energy is radiated, what was once a relatively strong magnetic field would become a weak one $\left(V_{A}<<c_{S}\right)$. Such turbulence in the RCS, therefore, could account for converting a strongly-magnetized jet into a weakly-magnetized one and for the tangled magnetic field that is often claimed in FSRQs [14, 23]. Furthermore, if the now largely de-magnetized jet retained much of its momentum through the RCS, not only would it still be a kinetic dominated flow, it also would behave very similarly to that of a non-magnetized supersonic flow. One way to convert a super-magnetosonic MHD jet into a supersonic hydrodynamic jet, then, is to dissipate and radiate the magnetic energy in a short period of time. The problem, of course, is to do this in the time it takes the super-magnetosonic flow to flow through the RCS, which is much less than the Alfvén crossing time.

In the Lazarian \& Vishniac picture of turbulent reconnection [19], for example, the speed of reconnection can be enhanced to a value $V_{\text {recon }} \sim V_{A} \mathcal{M}_{t}^{\lambda}$, where $\mathcal{M}_{t} \equiv V_{t} / c_{m s}$ is the turbulence Mach number and the exponent lies in the range $0.75 \leq \lambda \leq 2$. If the upper value $\lambda \sim 2$ applies, then the turbulence speed $V_{t}$ could be well below $V_{j}$ and yet the reconnection speed $\sim V_{j} \mathcal{M}_{t}^{2} / \mathcal{M}_{j}$ could be well above $V_{j}$. In this case there will be significant dissipation of the magnetic field before the jet passes completely through the RCS.

Much more analytic and semi-analytic work needs to be done besides these simple sums. However, this model for forming FR II jets can be tested with fairly straightforward resistive MHD simulations. Two, as well as three, dimensional simulations would be useful, and they could be set up in a manner similar to that in [8], [20], etc. Low-resistive control studies should be done first, and they should behave similarly to the earlier ideal MHD ones. Then the resistive dissipation (perhaps localized in the master recollimation shock in the simulation) should be increased to a value substantially larger than the general dissipation of the numerical scheme. The change in the properties of the post-RCS "nose-cone" jet as the dissipation increases are of greatest interest here. Early simulations of this type will show substantial thermal heating of the post-RCS jet and a marked decrease in the dominance of the magnetic field. In order to more accurately simulate what might happen in an actual FSRQ RCS, some (probably optically thin) radiative losses would need to be added to cool the post-RCS internal jet plasma in order to keep the flow supersonic.

The second, more speculative, part of the PhFH, then, is that more powerful black hole jets are actually able to dissipate most of their internal magnetic field in a strong RCS, thereby converting a strongly-magnetized $\left(V_{A}>>\right.$ $c_{S}$ ) flow into a weak one, thereby producing a stable, super(magneto)sonic, FR II-like jet within parsecs of the central BH.

\section{Discussion and Predictions}

\subsection{HST-1 in M87 as a Master RCS}

The feature HST-1 in the M87 jet has all the hallmarks of being the source's master recollimation shock. It remains 
stationary (at a speed of less than $0.25 c$ ); and it ejects relativistic jet components every few years, showing that it (not the core near the $\mathrm{BH}$ ) is the source of the superluminal components at $4-6 c[5,28,30]$. Furthermore, while the M87 jet clearly accelerates from the core up to HST-1, it then decelerates from superluminal to subluminal speeds beyond that feature. So, even though HST-1 ejects superluminal components, it is clear that beyond it the jet fails to maintain its super-(magneto)sonic character: it is at HST1 that the jet decides that it is not going to be an FR II source. The black hole mass of $\sim 6 \times 10^{9} M_{\odot}$ is $20-60$ times larger than the $1-3 \times 10^{8} M_{\odot}$ considered more typical of extragalactic radio sources.

\subsection{A Master RCS in Every Powerful Radio Jet}

The main prediction of the Phoenix Fire Hypothesis is that virtually every extragalactic radio sources (BL Lacs/FR Is and FSRQs/FR IIs alike) possess a master recollimation shock. And it is at this shock where the super-magnetosonic jet that emerges from the acceleration and collimation zone is reborn into either a transmagnetosonic, magnetically-dominated one (BL Lac/FR I) or into a super-sonic, plasma-pressure-dominated jet (FSRQ/FR II). If the formation of the master RCS is controlled by external processes in the galactic core ISM, then it is likely that RCSs in similar elliptical galaxies (with similar black hole masses) would occur at similar distances from the central hole (as measured in $r_{g}=$ $G M_{\bullet} / c^{2}$ ), regardless of whether they produce an FR I or FR II object. On the other hand, if RCS formation is controlled by internal processes in the jet itself, then it is possible that the RCSs in FSRQs and in BL Lac sources may occur at widely different numbers of $r_{g}$ from the BH. For example, perhaps very powerful (FSRQ/FR II) jets form an RCS much closer to the BH than a BL Lac with a similar BH mass.

An answer to this question may be provided by a very high resolution imaging and monitoring study with the VLBA of a sample containing nearby examples of both BL Lac and FSRQ objects, whose latter natures (along with whether each BL Lac is an HBL or LBL) have been demonstrated clearly from observations at non-radio wavelengths. We can estimate a typical core-RCS separation on the sky if we use HST-1 as a typical example and assume that the RCS distance from the central BH scales with the BH mass. This would give a typical core-RCS separation in a $1-3 \times 10^{8} M_{\odot} \mathrm{BH}$ system of $5-15 \mathrm{pc}$. At a nearby redshift of $z=0.05$ and a viewing angle of 5 degrees, the core-RCS separation on the sky would be of order $0.2-0.5$ mas. Monitoring over several years would be needed in order to establish a component as being the master RCS rather than just another superluminal one.

\subsection{Verifying that the PhFH Can Produce FSRQ/FR II Type Jets}

Save for the observational search for a master recollimation shock in FSRQ and/or FR II nuclei, most of the effort in testing the Phoenix Fire Hypothesis at this stage should be done with resistive numerical super-magnetosonic jet simulations, as discussed above. As discussed much earlier in the introduction, observations show that these sources behave like MHD flows in which the magnetic field does not play a dynamical role. The key goal, then, is to understand how what we believe to be a magneticallydominated ACZ can suddenly become plasma-pressuredominated and behave similarly to a regular supersonic hydrodynamic flow.

\subsection{Verifying that the PhFH Can Produce BL Lac/FR I Type Jets}

In this case we know much more about the type of postRCS jet flow that will be produced by a non-dissipative recollimation shock, because some of the necessary simulations already have been done. The key in this case, then, is to verify (1) that $3-\mathrm{D}$ non-dissipative simulations obtain results similar to 2-D ones, (2) that BL Lac sources (like M87) each have an RCS, and (3) that post-RCS jet properties in these sources are similar to those in the nondissipative simulations. These properties are potentially very rich in character and may afford further quantitative analysis of the internal nature of the BL Lac jet.

FSRQ-type jets typically should display only one kind of wave - a fast magnetosonic wave or shock, which travels along the jet axis at a speed $\gtrsim c_{m s}$. The other two possible waves (slow magnetosonic and Alfvén) will follow the magnetic field and, if that is tangled, they will be lost in the jet internal dynamics.

On the other hand, BL Lac jets, which we suggest here are ones whose strongly helical magnetic character survives passage through the master RCS, potentially could display the full range of MHD waves: a fast MHD wave or shock that travels along the jet axis at high speed, compressing the helical field along the way; a slow MHD wave/shock that travels at the local plasma sound speed or slower, compressing only the plasma itself, with a speed in the jet frame of only $\gtrsim c_{s}$ and an observed speed in the lab frame possibly not much more than $V_{j}$ itself; and an Alfvén wave (not shock) that travels along the jet axis at an intermediate speed given by $V_{A, Z}=B_{Z} /(4 \pi \rho)^{1 / 2}$. If an observer were lucky enough to simultaneously observe both moving components and Alfvén waves, then it may be possible to determine whether the moving components are slow or fast MHD waves/shocks. For example, if the component speeds were faster than the wave speed (fast MHD mode), then one may be able to derive the magnetic pitch angle by comparing $V_{A, Z}$ and $c_{m s} \approx V_{A}$. On the other hand, if the wave speed were faster than that of the components, then one could get a lower limit on the ratio $V_{A} / c_{S}$ (how much the jet internal plasma is magnetically dominated).

It should be realized, of course, that such an Alfvén wave probably will occur not in just one dimension transverse to the jet, but rather in both - that is, it very well could be a torsional Alfvén wave like that envisioned by Uchida and colleagues [38]. Indeed, at a redshift of 0.05 
-0.1 , at only a 5 degree angle to the line of sight, and for a smaller size black hole, the first several arcseconds of the M87 jet would correspond to a similar number of milliarcseconds in the core of a BL Lac source. So, the same type of helical kinks suggested in the M87 kiloparsec-scale jet may be visible in VLBI observations of BL Lac type blazars.

Nakamura has shown that the Poynting flux-dominated regions of the jet, which lie mainly in the submagnetosonic portion of the ACZ and in the post-RCS region, should be unstable to helical kink instabilities [29], and the jet probably will not be disrupted by these [24, 29]. So, the ACZ of any jet itself could develop helical kinks that ultimately move the master RCS around a bit from its quasi-stationary position. And, the post-RCS part of a BL Lac jet could locally develop helical kinks that may be observable.

\subsection{Component \#7 in BL Lac as a Master RCS}

The reader is encouraged to look at [6] and [7], in which evidence for both a master recollimation shock and transverse waves in the source BL Lacertae itself is presented.

\section{References}

[1] Algaba, J., Asada, K., \& Nakamura, M., this conference.

[2] Asada, K., Nakamura, M., Doi, A., Nagai, H., \& Inoue, M., Proc. IAU S275, 6, 198-199, (2011).

[3] Bicknell, G.V., Astroph. J. Supp. Ser., 101, 29-39, (1995).

[4] Blandford, R.D. \& Payne, D.G., Mon. Not. R. Astron. Soc., 199, 883-903, (1982).

[5] Cheung, C.C., Harris, D.E., \& Stawarz, Ł., Astroph. J., 663, L65-L68, (2007).

[6] Cohen, M., this conference.

[7] Cohen, M. and the MOJAVE collaboration, in preparation.

[8] Clarke, D.A., Norman, M.L., \& Burns, J.O., Astroph. J., 311, L63-L67, (1986).

[9] Fanaroff, B.L. \& Riley, J.M., Mon. Not. R. Astron. Soc., 167, 31-35, (1974).

[10] Gabuzda, D.C., Proc. Natl. Acad. Sci. USA, 92, 11393-11398 (1995).

[11] Gabuzda, D.C., this conference.

[12] Giroletti, M., Giovanni, G., Taylor, G., \& Falomo, R., Astroph. J., 636, 801-814 (2006).

[13] Gopal-Krisha \& Wiita, P. J., Astron. Astroph., 363, 507-516, (2000).

[14] Hughes, P.A., Aller, H.D., \& Aller, M.F., Astroph. J., 341, 54-67, (1989).
[15] Hughes, P.A., Aller, H.D., \& Aller, M.F., Astroph. J., 341, 68-79, (1989).

[16] Jackson, C.A. \& Wall, J.V., Mon. Not. R. Astron. Soc., 304, 160-174, (1999).

[17] Komissarov, S.S., Mon. Not. R. Astron. Soc., 308, 1069-1076, (1999).

[18] Krause, M. \& Camenzind, M., Astron. Astroph., 380, 789-804 (2001).

[19] Lazarian, A. \& Vishniac, E.T. Astroph. J., 517, 700718 (1999).

[20] Lind, K.R., Payne, D.G., Meier, D.L., \& Blandford, R.D., Astroph. J., 344, 89-103, (1989).

[21] Livio, M., Ogilvie, G.I., \& Pringle, J.E. Astroph. J., 512, 100-104, (1999).

[22] Lynden-Bell, D., Mon. Not. R. Astron. Soc., 279, 389-401, (1996).

[23] Marscher, A.P. \& Gear, W.K., Astroph. J., 298, 114127 (1985).

[24] McKinney, J.C. \& Blandford, R.D., Mon. Not. R. Astron. Soc., 394, L126-L130, (2009).

[25] Meier, D.L., Astroph. J., 522, 753-766, (1999).

[26] Meier, D.L., Black Hole Astrophysics: The Engine Paradigm (Springer-Praxis, Heidelberg, 2012).

[27] Meier, D.L., Edgington, P.G., Godon, P., Payne, D.G., \& Lind, K.R., Nature, 388, 350-352, (1997).

[28] Nakamura, M., Garofalo, D., \& Meier, D.L., Astroph. J., 721, 1783-1789, (2010).

[29] Nakamura, M. \& Meier, D.L., Astroph. J., 617, 123154, (2004).

[30] Nakamura, M. \& Meier, D.L., submitted (2013).

[31] Norman, M.L., Smarr, L., Winkler, K.-H. A., \& Smith, M.D., Astron. Astroph., 113, 285-302, (1982).

[32] Norman, M.L. \& Winkler, K.-H. A., Supersonic Jets, Los Alamos National Laboratory publication (1985).

[33] Polko, P., Meier, D.L., \& Markoff, S., Astroph. J., 723, 1343-1350, (2010).

[34] Polko, P., Meier, D.L., \& Markoff, S., Mon. Not. R. Astron. Soc., 428, 587-598, (2013).

[35] Polko, P., Meier, D.L., \& Markoff, S., Mon. Not. R. Astron. Soc., submitted (2013).

[36] Ustyugova, G.V., et al., Astroph. J., 541, L21-L24, (2000).

[37] Vlahakis, N., Tsinganos, K., Sauty, C., \& Trussoni, E., Mon. Not. R. Astron. Soc., 318, 417-428, (2000).

[38] Uchida, Y., Nakamura, M., Hirose, S., \& Uemura, S., Astroph. Sp. Sci., 264, 195-212 (1999).

[39] Werner, M., Murphy, D., Livingston, J., Gorjian, V., Jones, D., Meier, D., \& Lawrence, C., Astroph. J., 759, 86-107 (2012). 